\title{
Digital Holography Data Compression
}

\author{
Roberto Corda
}

\begin{abstract}
Digital holography processing is a research topic related to the development of novel visual immersive applications. The huge amount of information conveyed by a digital hologram and the different properties of holographic data with respect to conventional photographic data require a comprehension of the performances and limitations of current image and video standard techniques. This paper proposes an architecture for objective evaluation of the performances of the state-of-the-art compression techniques applied to digital holographic data.
\end{abstract}

Keywords - computer generated holography, compression, digital holography, JPEG 2000, HEVC.

\section{INTRODUCTION}

$\mathrm{S}$ YSTEMS for displaying content in three dimensions are the natural evolution of the traditional 2-D displays. Since the $20^{\text {th }}$ century, several approaches have been investigated for providing the observer with the perception of depth.

One of the most widespread techniques to achieve this goal is based on the stereoscopic principle [1], where two 2-D slightly different images are individually shown to the two eyes of the observer. Due to their operating principle, stereoscopic displays create a mismatch between the accommodation distance and the convergence distance, which can cause discomfort or sickness to the observer [2].

Holography is the only technique capable of providing the image with all the characteristics necessary for the observer to have a perception of the three-dimensional scene that is exactly the same as the reality [3]. It is based on the interference of electromagnetic waves that fall within the visible spectrum. Although there are several techniques for acquiring the hologram of a real object, a general setup includes one (or more, in the case of color acquisitions) coherent light generators, typically laser, a beam-splitter, and an acquisition plate.

The beam generated by the laser is divided into two parts by means of the beam-splitter, from which a reference wave and an object wave are originated. The reference wave directs itself towards the acquisition plate, while the object

Paper received April 26, 2019; revised May 24, 2019; accepted May 29, 2019. Date of publication July 31, 2019. The associate editor coordinating the review of this manuscript and approving it for publication was Prof. Irini Reljin.

This paper is revised and expanded version of the paper presented at the 26th Telecommunications Forum TELFOR 2018 [45].

This research activity has been partially funded within the Cagliari2020 project (MIUR, PON04a2 00381) and the DigitArch Cluster Top-Down project (POR FESR, 2014-2020).

Roberto Corda is with the University of Cagliari, Italy (email: r.corda@icloud.com). wave first hits the object, and successively it also goes towards the acquisition plate.

The interference between these two waves is recorded by the acquisition plate and constitutes what is called a hologram. Through this method it is possible to acquire not only information on the amplitude of the electromagnetic wave (as in the photograph), but also its phase. To obtain the holographic image, the hologram will have to be illuminated again with the reference wave, and this will project an image that is indistinguishable from the object originally acquired [4]. The acquisition of holograms with optical setups is a complex process, which requires specialized equipment and environments.

An alternative way is to use computerized methods, which numerically calculate the propagation of the electromagnetic field, simulating the generation and reconstruction processes. The computation of the so-called Computer Generated Hologram (CGH) takes place starting from a three-dimensional representation of a synthetic scene, typically modeled by polygons or point clouds.

In these cases, every primitive of the scene (point or polygon) is considered as an element that emits an electromagnetic wave, and the various contributions of the different primitives are combined in the hologram plane, in order to obtain the holographic representation of the whole scene [5].

Other basic representations are also possible, such as the ray-based model, in which the scene is represented as a set of light rays [6]-[30], or the layer-based model, in which the depth of the scene is represented by multiple layers: the contribution of each layer is propagated towards the hologram plane and added to the others in order to obtain the final hologram [5]. A further acquisition technique consists in combining different views of the same scene, acquired from different perspectives (multiviewpoint projections), which are then processed to obtain the final hologram [31]. Regardless of the method used, the computational complexity to create a CGH is typically high, so research in this field continues to be very active [32].

Holographic displays are obviously necessary to exploit the great potential of holography. To date, different types of holographic displays have been proposed [33], including head-mounted solutions [34], which are less penalized in terms of view angles and observation window dimensions, due to the limited display size and the short distance between the display and the observer's eyes. Indeed, it is expected that a head-mounted display will be the first type of holographic display that will become commercially available in the next few years. In contrast, high quality multi-user displays whose dimensions are comparable to current television sets (or bigger), are not supposed to be available for another decade [35]. 
Data compression is another fundamental challenge to be faced in order to facilitate the development and deployment of holographic applications. Typically, a hologram characterized by a high resolution and wide viewing angles, comprises a very large amount of data. It is essential to find a coding method that makes transmission, storage and processing less difficult.

In the literature there are several researches dedicated to the compression of the holographic data [36], [35]. Different types of lossless algorithms have been tested in [37]. In some cases, the coding techniques that have been used for holograms compression are the current standards for image and video compression [38], [39]. In some researches, new elements have been added to the compression process in order to improve performance [39][41], and also wavelet transforms have been proposed, different from the standard ones, such as the Gabor wavelet [42] and the Morlet wavelet [43].

Despite different data compression approaches have been proposed, no standard solution has been established so far, and further studies are needed to better understand the holographic data features. The ISO/IEC JPEG standardization committee has recently started an activity, named JPEG Pleno, with the aim to explore, among other challenges, the holographic data compression field [44].

The objective of this research is to perform a compression benchmark on $\mathrm{CGH}$ holographic data using JPEG 2000 and HEVC, expanding with respect to the previous research the modalities in which the holographic image quality is evaluated. Since the holographic image has the main characteristic of being three-dimensional, it is important to evaluate its quality taking this aspect into account, and this implies carrying out evaluations from different perspectives and reconstruction distances of the image. In order to simplify the reproducibility of the results, only publicly available CGH have been employed. With respect to the previous work [45], a more in-depth description of the proposed architecture is provided, including also new results regarding the experimental analysis that are here deeply analyzed.

This paper is structured as follows. In Section II an overview of the main digital hologram data representation forms is given. Section III describes the proposed architecture and the metrics used for evaluating the results. Section IV is dedicated to the description of the datasets, configuration of encoders, presentation of experimental results, and discussion. Finally, the conclusions are drawn in Section V.

\section{Digital HOlOGRAPHY OVERVIEW}

The hologram acquisition process consists in registering the interference fringe (fringe patterns) between the object wave and the reference wave. The hologram is then described by a complex waveform $U_{0}\left(x_{0}, y_{0}\right)$ that can be represented in several ways, depending also on the used acquisition technique. A common modality consists in describing the hologram in one of the equivalent complex number representations, namely the algebraic form and the polar form.
The algebraic form is composed of a real part and an imaginary part, described as:

$$
\begin{aligned}
U_{0}\left(x_{0}, y_{0}\right)= & \operatorname{real}\left(U_{0}\left(x_{0}, y_{0}\right)\right) \\
& +j \cdot \operatorname{imag}\left(U_{0}\left(x_{0}, y_{0}\right)\right),
\end{aligned}
$$

where $j$ denotes the imaginary unit.

The polar form is composed of an amplitude part and a phase part, described as:

$$
U_{0}\left(x_{0}, y_{0}\right)=A\left(x_{0}, y_{0}\right) e^{j \phi\left(x_{0}, y_{0}\right)} .
$$

When the acquisition technique is based on the phaseshifting method, the complex wave is obtained by several (usually between two and four) interferograms that are acquired by changing the wave's phase for each acquisition.

For example, assuming four acquisition steps with phase shift equal to $\pi / 2$, the complex wave is represented as:

$$
\begin{aligned}
U_{0}\left(x_{0}, y_{0}\right)= & \frac{1}{4 U_{r}^{*}}\left(\left(\left(I\left(x_{0}, y_{0} ; 0\right)-I\left(x_{0}, y_{0} ; \pi\right)\right)\right.\right. \\
& \left.-j\left(I\left(x_{0}, y_{0} ; \frac{\pi}{2}\right)-I\left(x_{0}, y_{0} ; \frac{3 \pi}{2}\right)\right)\right),
\end{aligned}
$$

where $U_{r}$ represents the reference wave, the symbol * the complex conjugate, and $I$ represents the interferogram.

Other acquisition techniques are based on capturing three inteferograms. In this case the complex wave is represented by:

$$
\begin{aligned}
U_{0}\left(x_{0}, y_{0}\right)= & I\left(x_{0}, y_{0} ; 0\right)+e^{-j 2 \pi / 3} I\left(x_{0}, y_{0} ;-\frac{2 \pi}{3}\right) \\
& +e^{j 2 \pi / 3} I\left(x_{0}, y_{0} ; \frac{2 \pi}{3}\right) .
\end{aligned}
$$

Other methods are based on the acquisition of two interferograms and are named two steps or quadrature phase shifting holography methods [46].

\section{PROPOSED ARCHITECTURE}

A common representation of the holographic data is a matrix of complex numbers, a completely different form compared to conventional images or video contents. Several processing steps are required for converting the hologram data in a data format compliant with the input format of image and video codecs. The compression and quality evaluation architecture used in this work is shown in Fig. 1 and it is discussed in the remainder of this section.

The Complex raw data block represents the holographic data composed of one complex matrix (for grayscale holograms) or three complex matrices (for RGB color holograms). These data are the input of the Range mapping \& Quantization block. In this block, the input data are mapped on a range $\left[0,\left(2^{\mathrm{n}}-1\right)\right]$, controlled by the number of quantization bits $n$, which is an input parameter for the block. The output of the block has two components (F1 and F2), represented with $n$ bit per sample (bps), which correspond to the two parts of the complex representation: the real and imaginary parts or the amplitude and phase parts. In these experiments, $n=8 \mathrm{bps}$ has been employed.

The two parts $F 1$ and $F 2$ are independently encoded and decoded in the Coding and Decoding blocks respectively. At this stage, the comparison in the so-called Hologram Domain is performed: the output signals ( $F 1 \mathrm{dec}$. and 


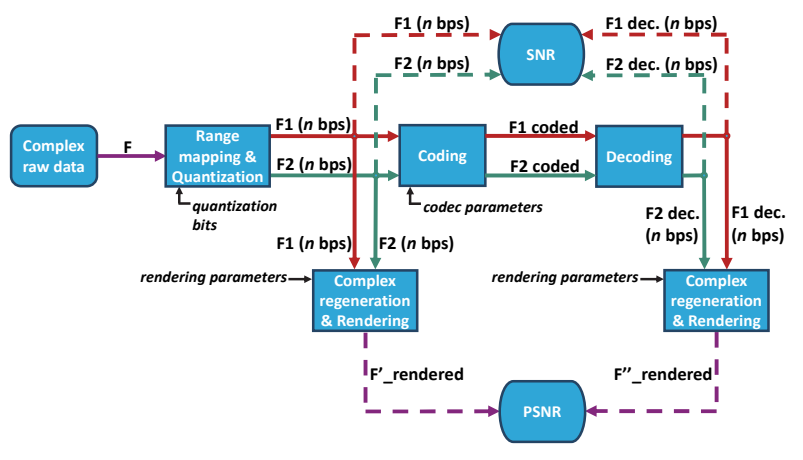

Fig. 1. Proposed compression architecture.

$F 2$ dec.) are compared with the reference signals ( $F 1$ and $F 2$ ) in terms of signal-to-noise ratio (SNR), as defined in [47]:

$$
S N R=10 \log _{10} \frac{\sum_{x=0}^{M-1} \sum_{y=0}^{N-1}[r(x, y)]^{2}}{\sum_{x=0}^{M-1} \sum_{y=0}^{N-1}[r(x, y)-t(x, y)]^{2}},
$$

where $r(x, y)$ denotes the reference samples while $t(x, y)$ denotes the test samples (i.e. the decoded samples).

In the Complex regeneration \& Rendering block, the reference and test components are mapped again into their original data ranges and merged in a single complex matrix in order to be ready for the image reconstruction step.

The Angular Spectrum Method (ASM) has been used for the image reconstruction, followed by a rendering process. After the image reconstruction, the image quality evaluation has been performed, in the so-called Reconstructed Domain, comparing the reference image $F$, rendered with the test image $F$, rendered with the PSNR metric:

$$
P S N R=10 \log _{10} \frac{\left(2^{n}-1\right)^{2}}{\frac{1}{M N} \sum_{x=0}^{M-1} \sum_{y=0}^{N-1}[r(x, y)-t(x, y)]^{2}} .
$$

The $F$ rendered image is generated from the data that have not been compressed, while the $F$, rendered image is generated from the data that have been previously compressed.

The PSNR has been chosen for the evaluation in the Reconstructed Domain because the data under comparison is the final image that is shown to the user. In contrast, in the Hologram Domain, the data that constitutes the hologram are compared, and thus the SNR metric has been employed. It should be pointed out that currently no standard metrics or evaluation methods have been established for quality evaluation on holographic contents.

\section{EXPERIMENTAL ANALYSIS}

\section{A. Test modalities and Datasets}

In this work, the PSNR has been evaluated on two distinct scenarios. The first scenario evaluates the PSNR as the reconstruction distance changes, keeping a fixed perspective on the scene. For this experiment, the central perspective has been chosen. For each level of compression, the PSNR has been calculated by performing 30 different reconstructions at 30 different distances, chosen in such a way as to uniformly cover the entire range of distances allowed by the sample under test. The second scenario instead, evaluates the PSNR as the perspective on the scene changes. For each compression level, 36 different reconstructions from 36 different perspectives have been performed, uniformly distributed within the range of allowed perspectives.

The encoders that have been used for the compression analysis are the HEVC reference software [48] version 16.18, and the JPEG 2000 Kakadu software [49] version 7.10.2. For each codec, nine different compression levels have been selected. The HEVC compression quality has been controlled using the quantization parameter with the values $q=\{0,2,4,7,10,20,30,40,51\}$, where $q=0$ is the best quality, while $q=51$ is the worst quality. The JPEG 2000 quality has been controlled using the rate parameter that allows to declare a target bitrate for the compressed data. In order to ensure a fair comparison between the two codecs, the JPEG 2000 rate has been set equal to the output data bitrate of HEVC coding that has been previously performed.

The two samples under test are reconstructed using the ASM, but the subsequent rendering process is slightly different depending on the chosen sample. The first sample belongs to the Interfere II dataset. As suggested by the authors in [50], the observation window has resolution of $2048 \times 2048$, and it is apodized using a 2-D Hanning window before the reconstruction process. In order to reduce the speckle noise, the final image is the average of three reconstructions performed at three different wavelengths $(633 \mathrm{~nm}, 632.9 \mathrm{~nm}$, and $633.1 \mathrm{~nm})$. This technique has been chosen because it has higher computational efficiency than other techniques for reducing the speckle effect. The second sample belongs to the B-com's dataset [31]. The framework proposed in [51] has been employed for the rendering process: an observation window of $2048 \times 2048$ has been extracted and apodized. After the reconstruction, the image has been enhanced through a percentile clipping, followed by a linear histogram equalization process.

The dataset Interfere II [50] presents holograms created using a method that supports occlusion and simulation of diffuse reflection from surfaces. All the holograms are monochromatic, generated from point clouds. The pixel pitch is $1 \mu \mathrm{m}$ with resolution of $8192 \times 8192$, and fullparallax support. The hologram selected from this dataset is Ball $8 K D$. The other sample under test is Specular Car $8 \mathrm{~K}$ from B-com's dataset. This hologram has been generated using the algorithm described in [31], which supports specular reflections reproduction, occlusions and fullparallax. The source data are multiview-plus-depth. Specular Car $8 K$ is a color hologram, with pixel pitch of $0.4 \mu \mathrm{m}$ and resolution of $8192 \times 8192$.

\section{B. Results}

For what concerns the Hologram Domain, the results of Ball $8 K D$ are shown in Fig. 2. The Fig. 2 (left) shows the real part while Fig. 2 (right) shows the imaginary part. The curves show that HEVC provides better results than JPEG 2000 at medium-low bitrates. For higher bitrates it is the JPEG 2000 that outperforms HEVC. For both the real and imaginary parts, the lowest bitrate point of HEVC is not 

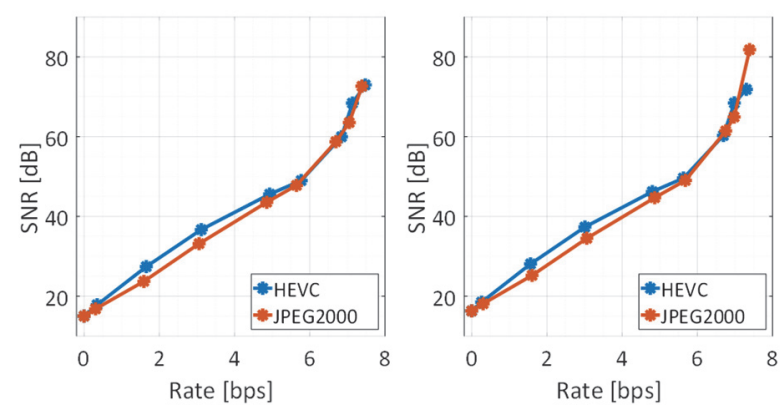

Fig. 2. Ball $8 K D$ SNR result: real part (left) and imaginary part (right).
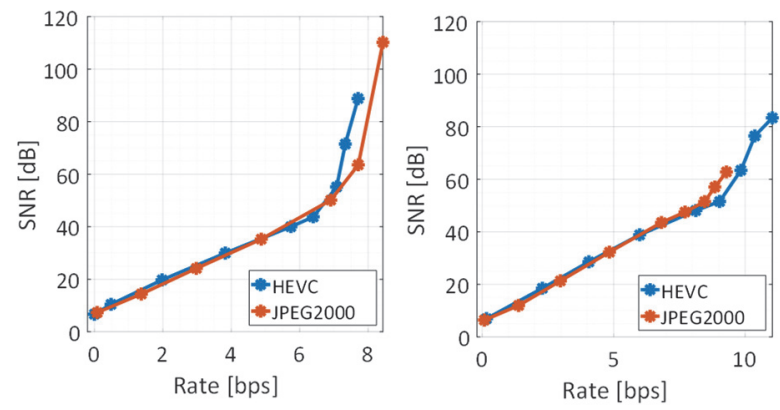

Fig. 3. Ball $8 K D$ SNR results: amplitude (left) and phase (right).

visible because it is the same of JPEG 2000, both corresponding to $14 \mathrm{~dB}$. For what concerns the polar representation, Fig. 3 (left) shows the amplitude results while Fig. 3 (right) the phase results. At medium-low bitrates the difference between the two codecs is marginal and the SNR results are lower if compared with the algebraic representation. For bitrates higher than $8 \mathrm{bps}$, the JPEG 2000 obtains a lossless compression: only 7 orange compression points are visible in Fig. 3 (left). The phase compression (Fig. 3, right) at low bitrates gives SNR results similar to amplitude compression, but JPEG 2000 outperforms HEVC from $7 \mathrm{bps}$ to $9.5 \mathrm{bps}$.

The results of Specular Car $8 \mathrm{~K}$ bring to similar results for both real and imaginary components, showed in Fig. 4 (left) and in Fig. 4 (right) respectively. At low bitrates HEVC outperforms JPEG 2000, while for bitrates higher than nearly 12 bps it is JPEG 2000 that gives better performances. For what concerns the polar representation (Fig. 5), the results are again similar to the previous sample: the two codecs show similar performances, lower than the algebraic representation.

For what concerns the Reconstructed Domain, each compression point in Figs. 6-9 represents the mean PSNR, while its standard deviation is indicated by a vertical interval. In the experiments with the change in reconstruction distance, every point in the graph is the mean PSNR of 30 image reconstructions at 30 different distances, while in the experiments with the change of perspective every point in the graphs is the mean PSNR of 36 image reconstructions from 36 different perspectives. In order to quantify the performance difference between the two codecs, also the Bjøntegaard rate gain (BD-Rate) and distortion gain (BD-PSNR) of HEVC over JPEG 2000 are
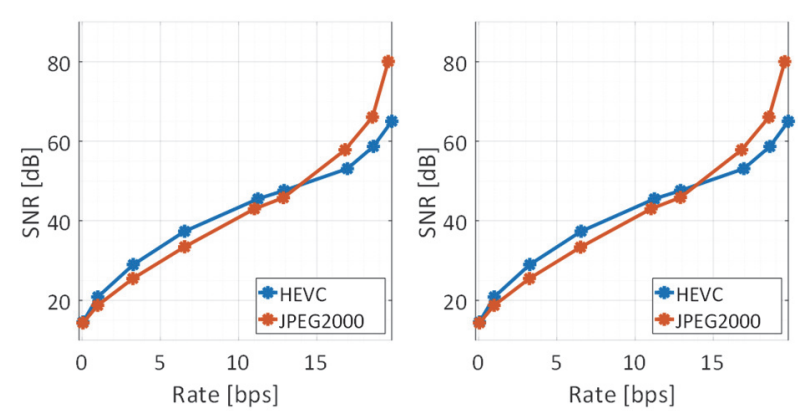

Fig. 4. Specular Car $8 K$ SNR results: real part (left) and imaginary part (right).
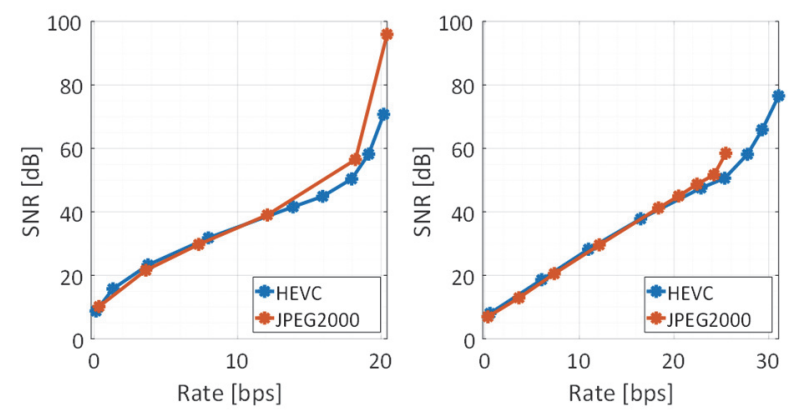

Fig. 5. Specular Car $8 K$ SNR results: amplitude (left) and phase (right).

provided in this domain. Fig. 6 shows the results for Ball $8 K D$ in algebraic form, with the change in perspective (left) and in reconstruction distance (right). It can be noted that with the change in perspective the PSNR variance is high (up to $\pm 5 \mathrm{~dB}$ ) at high bitrates. The BD-PSNR is $3.13 \mathrm{~dB}$ with a BD-Rate of $-15.02 \%$. With the change in reconstruction distance, the variations are around $\pm 2 \mathrm{~dB}$, or lower, at any compression level, while BD-PSNR is $3.45 \mathrm{~dB}$ and BD-Rate is $-25.27 \%$. In Fig. 7 the results of Ball $8 K D$ in polar form are shown. It can be noted that at equal bitrates, often the PSNR values are slightly lower than in the algebraic case. The mean PSNR variations are higher at high bitrates, especially for HEVC, with the change in the perspective (Fig. 7, left), where variations up to $\pm 4 \mathrm{~dB}$ can be noted. The BD-PSNR is $7.5 \mathrm{~dB}$ with a BD-Rate of $-27.51 \%$. A similar scenario can be seen with the change in reconstruction distance in Fig. 7 (right), in which the BDPSNR and BD-Rate are respectively $5.67 \mathrm{~dB}$ and $-19.02 \%$. Both when the perspective changes and the reconstruction distance varies, JPEG 2000 is the codec that is most affected by the polar representation, especially at low bitrates.

In Fig. 8 the mean PSNR results of Specular Car $8 \mathrm{~K}$ in algebraic form are reported. In both graphs we can see a codec behavior similar to the previous grayscale sample, but with reduced PSNR variations, especially with the change in perspective with the HEVC compression (Fig. 8, left). The BD-PSNR is $0.75 \mathrm{~dB}$ with a BD-Rate of $-26.07 \%$. The mean PSNR values with the change in reconstruction distance (Fig.8, right) are always inside the $\pm 2 \mathrm{~dB}$ range. The overall trend is similar to the previous case: this fact is also confirmed by the Bjøntegaard metrics that show a BDPSNR of $1.21 \mathrm{~dB}$ and a BD-Rate of $-28.01 \%$. Finally, the mean PSNR results of Specular Car $8 K$ in polar form 

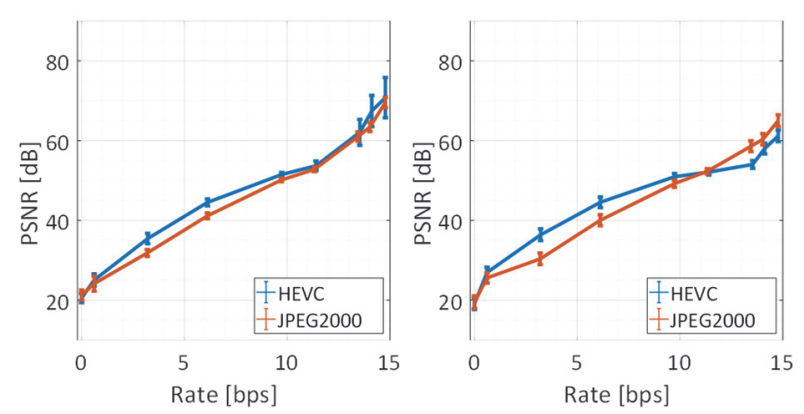

Fig. 6 Ball $8 K D$, algebraic form: PSNR results with the change in perspective (left) and in reconstruction distance (right)
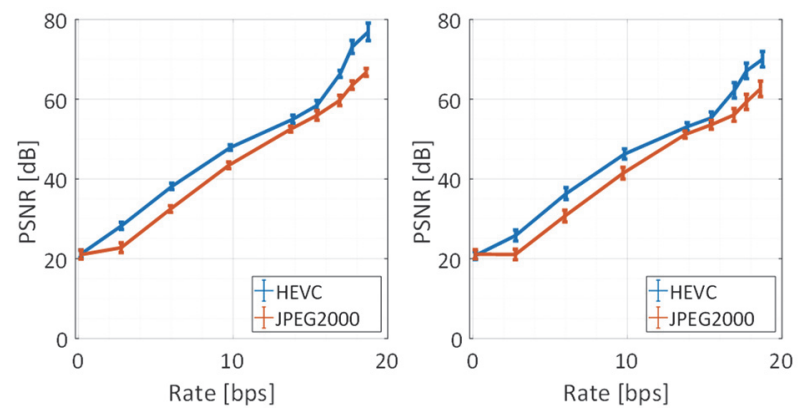

Fig. 7 Ball $8 K D$, polar form: PSNR results with the change in perspective (left) and in reconstruction distance (right)

are showed in Fig. 9. The mean PSNR variations with the change in perspective (Fig. 9, left) are negligible, being at most around $\pm 1 \mathrm{~dB}$. In this case the BD-PSNR is equal to $5.63 \mathrm{~dB}$ with a BD-Rate of $-34.58 \%$. The PSNR variations with the change in reconstruction distance (Fig. 9, right) are also limited, around to $\pm 2 \mathrm{~dB}$ and similar to the algebraic representation. The BD-PSNR is $4.92 \mathrm{~dB}$ with a BD-Rate of $-40.14 \%$. The experiments in the Reconstructed Domain show that HEVC provides the overall better performance, with BD-rate savings up to $40 \%$ with respect to JPEG 2000. One last aspect to note is how, in the polar representation, if forced at very high output bitrates, the codecs produce an output with a bitrate higher than the input: in the case of Ball $8 K D$, the $16 b p s$ are exceeded, while in the case of Specular Car $8 K$ the $48 \mathrm{bps}$ are exceeded. This fact is mainly caused by the phase data, as can also be verified by examining the previous curves in the Hologram Domain (Fig.3 and Fig. 5). This further highlights the difficulty for standard codecs of obtaining good results with this type of data.

\section{CONCLUSIONS}

Digital holography compression performance of state ofthe-art image and video standards, namely HEVC and JPEG 2000 , has been evaluated. The objective quality evaluation has been performed using the SNR metric for the holographic data prior to the reconstruction, and using the PSNR metric for the reconstructed holographic image. In particular, the PSNR metric has been computed both for the holographic data prior to the reconstruction, and using the PSNR metric for the reconstructed holographic image.
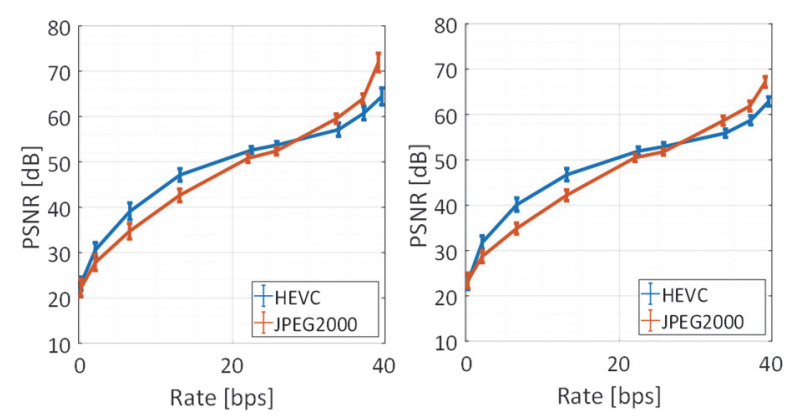

Fig. 8. Specular Car $8 K$, algebraic form: PSNR results with the change in perspective (left) and in reconstruction distance (right).
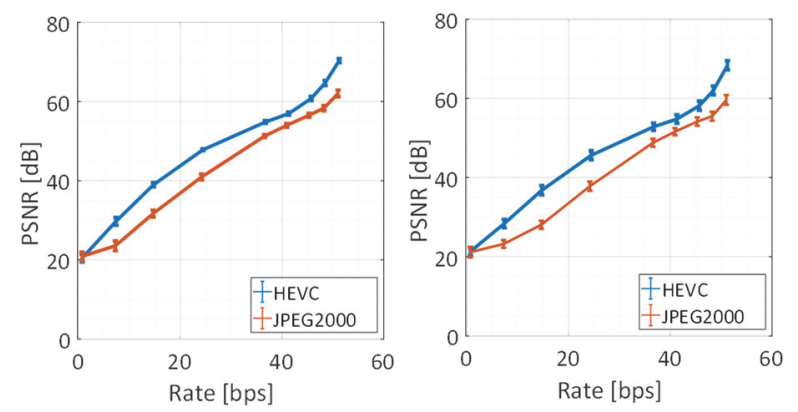

Fig. 9. Specular Car $8 K$, polar form: PSNR results with the change in perspective (left) and in reconstruction distance (right).

In particular, the PSNR metric has been computed both for perspective and reconstruction distance variations. The poor obtained results in terms of compression ratio with respect to compression ratios achievable with regular images, confirm the necessity for novel encoders designed for the characteristic of the holographic data.

\section{REFERENCES}

[1] J. Konrad and M. Halle, "3-D displays and signal processing," IEEE Signal Processing Magazine, vol. 24, no. 6, pp. 97-111, 2007.

[2] D. M. Hoffman, A. R. Girshick, K. Akeley, and M. S. Banks, "Vergence-accommodation conflicts hinder visual performance and cause visual fatigue," Journal of vision, vol. 8, no. 3, pp. 33-33, 2008

[3] C. Slinger, C. Cameron, and M. Stanley, "Computer-generated holography as a generic display technology," Computer, vol. 38, no. 8, pp. 46-53, 2005.

[4] U. Schnars and W. P. Jüptner, "Digital recording and numerical reconstruction of holograms," Measurement science and technology, vol. 13, no. 9, p. R85, 2002.

[5] J.-H. Park, "Recent progress in computer-generated holography for three-dimensional scenes," Journal of Information Display, vol. 18, no. 1, pp. 1-12, 2017.

[6] C. Perra, "On the coding of plenoptic raw images," in $22^{\text {nd }}$ Telecommunications forum (TELFOR), Belgrade, Serbia, November 2014.

[7] C. Perra, F. Murgia, and D. Giusto, "An analysis of 3D point cloud reconstruction from light field images," in International Conference on Image Processing Theory, Tools and Applications, Oulu, Finland, December 2016.

[8] C. Perra, "Re-encoding JPEG images for smart phone applications," in 21st Telecommunications Forum (TELFOR), Belgrade, Serbia, November 2013.

[9] C. Perra, W. Song, and A. Liotta, "Effects of light field subsampling on the quality of experience in refocusing applications," in 2018 Tenth International Conference on Quality of Multimedia Experience, May 2018. 
[10] C. Perra, "Assessing the quality of experience in viewing rendered decompressed light fields," Multimedia Tools and Applications, vol. 77, no. 16, Aug 2018.

[11] M. T. Vega, C. Perra, and A. Liotta, "Resilience of video streaming services to network impairments," IEEE Transactions on Broadcasting, vol. 64, no. 2, June 2018.

[12] M. T. Vega, C. Perra, F. D. Turck, and A. Liotta, “A review of predictive quality of experience management in video streaming services," IEEE Transactions on Broadcasting, vol. 64, no. 2, June 2018.

[13] C. Perra and D. Giusto, “A framework for view progressive coding of light field," in 2018 IEEE International Conference on Consumer Electronics (ICCE), Jan 2018.

[14] C. Perra and D. D. Giusto, "Light field compression on sliced lenslet array," International Journal of Internet Technology and Secured Transactions, vol. 8, no. 1, 2018.

[15] C. Perra and D. Giusto, "Raw light field image compression of sliced lenslet array," in 2017 IEEE International Symposium on Broadband Multimedia Systems and Broadcasting (BMSB), June 2017.

[16] C. Perra and D. Giusto, "JPEG 2000 compression of unfocused light field images based on lenslet array slicing," in 2017 IEEE International Conference on Consumer Electronics (ICCE), Jan 2017.

[17] C. Perra, "Light field coding based on flexible view ordering for unfocused plenoptic camera images," International Journal of Applied Engineering Research, vol. 12, no. 21, pp. 10 563-10 569, 2017.

[18] C. Perra and P. Assuncao, "High efficiency coding of light field images based on tiling and pseudo-temporal data arrangement," in IEEE International Conference on Multimedia and Expo, Seattle, USA, July 2016.

[19] A. Vieira, H. Duarte, C. Perra, L. Tavora, and P. Assuncao, "Data formats for high efficiency coding of lytro-illum light fields," in 2015 International Conference on Image Processing Theory, Tools and Applications (IPTA), Nov 2015.

[20] C. Perra, "Lossless plenoptic image compression using adaptive block differential prediction," in IEEE 40th International Conference on Acoustics, Speech and Signal Processing, Brisbane, Australia, April 2015.

[21] C. Perra, "A framework for user control over media data based on a trusted point," in 2015 IEEE International Conference on Consumer Electronics (ICCE), Jan 2015.

[22] C. Perra, P. A. Pes, and D. D. Giusto, "High-frequency error recovery in JPEG XR coded images," in 18th IEEE International Conference on Image Processing, Sept 2011.

[23] C. Perra, "Wireless cooperative access to images," in $20139^{\text {th }}$ International Wireless Communications and Mobile Computing Conference, July 2013.

[24] R. Corda, C. Perra, "A dataset of hologram reconstructions at different distances and viewpoints for quality evaluation," in 2019 Tenth International Conference on Quality of Multimedia Experience, June 2019.

[25] C. Perra, D. Giusto, "An analysis of HEVC compression for light field image refocusing applications", in 2018 IEEE International Conference on Communications and Electronics (ICCE), Jan 2018.

[26] F. Murgia, C. Perra, and D. Giusto, "3D point cloud reconstruction from single plenoptic image," Telfor Journal, vol. 8, no. 1, 2016.

[27] B. Saba, C. Perra, and D. D. Giusto, "Interactive image viewing in mobile devices based on JPEG XR," in International Conference on Mobile Multimedia Communications. Springer, 2011, pp. 227-241.

[28] M. Fadda, M. Murroni, C. Perra, and V. Popescu, "TV white spaces exploitation for multimedia signal distribution," Signal Processing: Image Communication, vol. 27, no. 8, pp. 893-899, 2012.

[29] C. Perra, "A framework for the development of sustainable urban mobility applications," in 2016 24th Telecommunications Forum, Nov 2016, pp. 1-4

[30] C. Perra, "Light field image compression based on preprocessing and high efficiency coding," in 2016 24th Telecommunications Forum, Nov 2016, pp. 1-4.

[31] A. Gilles, P. Gioia, R. Cozot, and L. Morin, "Computer generated hologram from multiview-plus-depth data considering specular reflections," in Multimedia \& Expo Workshops (ICMEW), 2016 IEEE International Conference on. IEEE, 2016, pp. 1-6.
[32] T. Nishitsuji, T. Shimobaba, T. Kakue, and T. Ito, "Review of fast calculation techniques for computer-generated holograms with the point-light-source-based model," IEEE Transactions on Industrial Informatics, vol. 13, no. 5, pp. 2447-2454, 2017.

[33] Y. Pan, J. Liu, X. Li, and Y. Wang, "A review of dynamic holographic three-dimensional display: Algorithms, devices, and systems," IEEE Transactions on Industrial Informatics, vol. 12, no. 4, pp. 1599-1610, 2016

[34] A. Maimone, A. Georgiou, and J. S. Kollin, "Holographic near-eye displays for virtual and augmented reality," ACM Transactions on Graphics (TOG), vol. 36, no. 4, p. 85, 2017.

[35] D. Blinder, A. Ahar, S. Bettens, T. Birnbaum, A. Symeonidou, H. Ottevaere, C. Schretter, and P. Schelkens, "Signal processing challenges for digital holographic video display systems," Signal Processing: Image Communication, vol. 70, pp. 114-130, 2019.

[36] F. Dufaux, Y. Xing, B. Pesquet-Popescu, and P. Schelkens, "Compression of digital holographic data: an overview," in Applications of Digital Image Processing XXXVIII, vol. 9599. International Society for Optics and Photonics, 2015, p. 95990I.

[37] T. J. Naughton, Y. Frauel, B. Javidi, and E. Tajahuerce, "Compression of digital holograms for three-dimensional object recognition," in Algorithms and Systems for Optical Information Processing $V$, vol. 4471. International Society for Optics and Photonics, 2001, pp. 280-290.

[38] J. Peixeiro, C. Brites, J. Ascenso, and F. Pereira, "Digital holography: benchmarking coding standards and representation formats," in 2016 IEEE International Conference on Multimedia and Expo (ICME). IEEE, 2016, pp. 1-6.

[39] J. P. Peixeiro, C. Brites, J. Ascenso, and F. Pereira, "Holographic data coding: Benchmarking and extending HEVC with adapted transforms," IEEE Transactions on Multimedia, vol. 20, no. 2, pp. 282-297, 2018.

[40] D. Blinder, T. Bruylants, H. Ottevaere, A. Munteanu, and P. Schelkens, "JPEG 2000-based compression of fringe patterns for digital holographic microscopy," Optical Engineering, vol. 53, no. 12, p. 123102, 2014.

[41] Y.-H. Seo, H.-J. Choi, and D.-W. Kim, "3D scanning-based compression technique for digital hologram video," Signal Processing: Image Communication, vol. 22, no. 2, pp. 144-156, 2007.

[42] K. Viswanathan, P. Gioia, and L. Morin, "Wavelet compression of digital holograms: towards a view-dependent framework," in Applications of Digital Image Processing XXXVI, vol. 8856. International Society for Optics and Photonics, 2013, p. 88561N.

[43] K. Viswanathan, P. Gioia, and L. Morin, "Morlet wavelet transformed holograms for numerical adaptive view-based reconstruction," in Optics and Photonics for Information Processing VIII, vol. 9216. International Society for Optics and Photonics, 2014, p. $92160 \mathrm{G}$.

[44] P. Schelkens, T. Ebrahimi, A. Gilles, P. Gioia, K.-J. Oh, F. Pereira, C. Perra, and A. M. Pinheiro, "JPEG Pleno: Providing representation interoperability for holographic applications and devices." ETRI Journal, vol. 41, no. 1, 2019.

[45] R. Corda, "Digital holography data compression," in 2018 26th Telecommunications Forum (TELFOR). IEEE, 2018, pp. 1-4.

[46] P. W. M. Tsang and T.-C. Poon, "Review on the state-of-the-art technologies for acquisition and display of digital holograms," IEEE Transactions on Industrial Informatics, vol. 12, no. 3, pp. 886-901, 2016.

[47] R. Gonzalez and R. Woods, Digital Image Processing. Pearson/Prentice Hall, 2008 pp. 374-379.

[48] "HEVC Software," [Online]. Available: https://hevc.hhi.fraunhofer.de/

[49] "Kakadu software,"[Online]. Available: http://kakadusoftware.com/

[50] A. Symeonidou, D. Blinder, A. Ahar, C. Schretter, A. Munteanu, and P. Schelkens, "Speckle noise reduction for computer generated holograms of objects with diffuse surfaces," in Optics, Photonics and Digital Technologies for Imaging Applications IV, vol. 9896. International Society for Optics and Photonics, 2016, p. 98960F.

[51] A. Symeonidou, D. Blinder, and P. Schelkens, "Colour computer generated holography for point clouds utilizing the phong illumination model," Optics express, vol. 26, no. 8, pp. $10282-10$ $298,2018$. 